\title{
3D numerical simulations of the Rossby wave instability
}

Héloïse Méheut

APC - Univ. Paris Diderot

E-mail: hmeheut@apc.univ-paris7.fr

\section{Fabien Casse}

APC - Univ. Paris Diderot

\section{Peggy Varnière}

APC - Univ. Paris Diderot

\section{Michel Tagger}

LPCE Orléans

Hydrodynamical or MHD instabilities have been proposed as explanations for the quasi-periodic oscillations observed in microquasars. One of them is the Rossby wave instability that have been studied in the case of an accretion disc to explain accretion in a non-magnetised disc (Lovelace, 1999). So far all numerical studies regarding the non-linear behaviour of this instability have been done using a two dimensional framework $(r, \theta)$, here 3D numerical simulations of the instability are presented.

VII Microquasar Workshop: Microquasars and Beyond September 1 - 5, 2008

Foca, Izmir, Turkey 


\section{Introduction}

The high frequency quasi-periodic oscillations (HF QPO) observed in microquasars may be explained by the development of a Rossby wave instability (RWI) at the marginally stable orbit (MSO) of the accretion disc (Tagger \& Varniere, 2006). Analytical and numerical studies of this instability have been achieved in the sole 2D context ( $\mathrm{Li}$ et al., 2000, 2001), without taking into account the vertical structure of the disc that can influence the characteristics of the instability and therefore the QPO frequency. Here we present 3D numerical simulations of the Rossby wave instability, and we show how the instability is unambiguously identified.

\section{The Rossby wave instability}

The Rossby wave instability was introduced by Lovelace et al. (1999) as a solution for the angular momentum transport in a (un-)magnetised disc. The hydrodynamical instability occurs when the radial profile of the specific vorticity shows a local extremum. The specific vorticity is the ratio between the vertical part of the velocity curl and the disc surface density. In a disc surrounding a compact object, the specific vorticity is naturally extremum close to the marginally stable orbit (MSO).

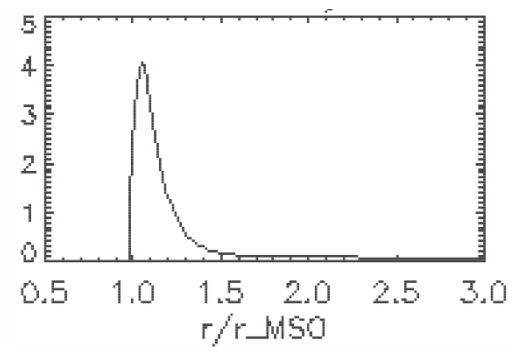

Figure 1: Radial profile of the specific vorticity in a disc with a usual surface density profile and a pseudonewtonian potential. $r_{M S O}$ is the radius of the marginally stable orbit.

The RWI is a non-axisymetric instability characterised by the formation of a Rossby vortex at the position of the extremum of the specific vorticity and density waves outside the vortex zone. Rossby vortices are usually discussed in meteorology and planetary atmosphere in general, the Jupiter great red spot is an example (fig 2). Differential rotation is also responsible for the coiling of the compressional waves into spirals, as in spiral galaxies (fig 2).

\section{Numerical setup}

The 3D simulations of the RWI were performed by the mean of the hydrodynamical module of the versatile advection code (VAC) code (Toth, 1996) solving the adiabatic equations. The resolution in cylindrical coordinates $(r, \theta, z)$ is $300 \times 256 \times 32$ or 64 cells on the computational domain $[0.8,10] \times.[0 ., 2 \pi] \times[0 ., 0.8]$ where $r=1$ correspond to $r=r_{M S O}$. The initial density profile is a usual profile with a surface density varying as $r^{-1 / 2}$ where we add an overdensity (a bump) at a radius of $3 r_{M S O}$ in order to satisfy the instability condition. 

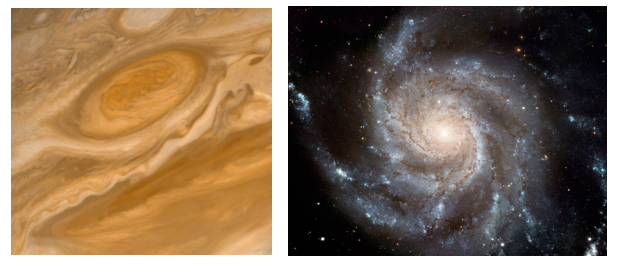

Figure 2: Right: Jupiter great red spot is an example of a Rossby vortex. Left: The arms of spiral galaxies are density waves occurring in a differentially rotating disc.
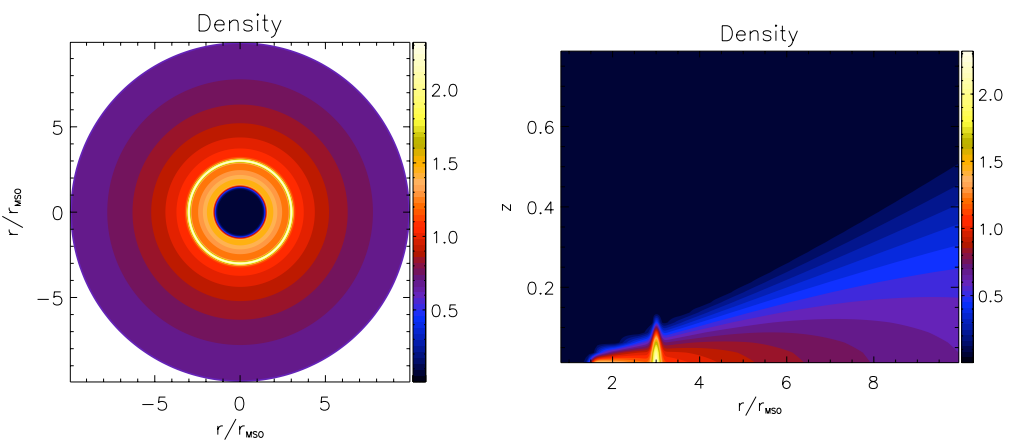

Figure 3: Initial density profile of the disc. The radius is given in function of the inner edge of the disc placed at $r=1$. Left: cross-section at the disc midplane. Right: vertical cross-section of the disc.

The radial velocity and vertical density profile were chosen in order for the disc to be in equilibrium. The axisymmetry of the disc is broken due to small perturbations of the radial velocity that are of the order of $10^{-4}$ times the local keplerian velocity. The instability grows exponentially from these initial perturbations.

\section{RWI identification}

The instability can be identified thanks to its two components, vortices and density waves, that appeared clearly in the simulations. Those two components can be seen on the density profile on figure 4.1. In the following plots, we display the physical quantities once have been subtracted the axisymmetric component of each quantity.

\subsection{Density waves}

The density waves are clearly identified when plotting the compressible part of the velocity $(\nabla . v)$. An interesting point is that the bump zone is known to be a forbidden zone regarding the density waves and it is clear on the left plot of fig 4.1 that those waves are only seen outside this zone.

\subsection{Vortices}

The vortices can be observed by plotting the vorticity of the stream that is the vertical pro- 

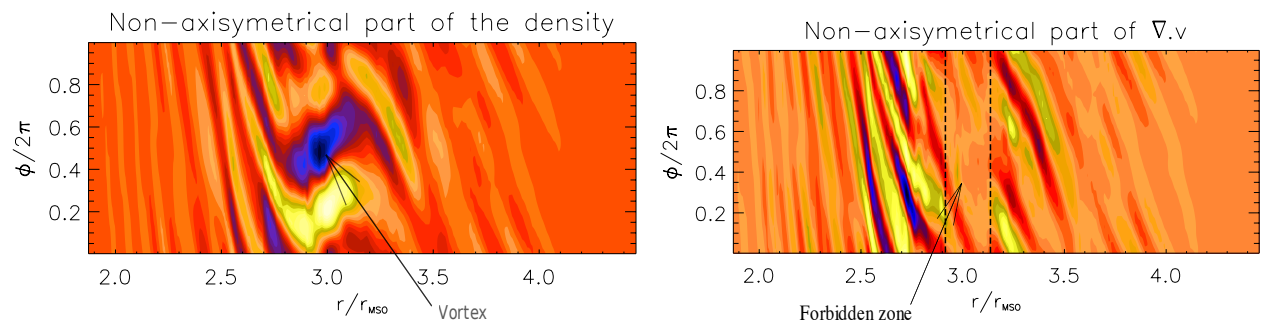

Figure 4: The two plots are a projection of the disc in a Cartesian grid, where the radius of the disc is in abscissa and the $\phi$ angle is in ordinate. The plots display $(r, \theta)$ cross-section of the disc between $\mathrm{r}=1.8$ and 4.2 . Left: Non-axisymetrical part of the density of the disc, the two parts of the instability can be seen: the vortex and the acoustic waves. Right: The plot of the non-axisymetrical part of $\nabla . v$ shows the compressible waves that are localised outside the bump zone.

jection of $\nabla \times v$. The exact location of the vortices can be confirmed by the velocity stream lines.

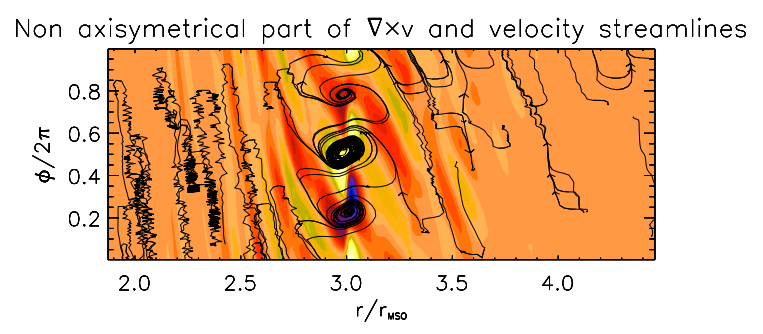

Figure 5: The same projection as fig. 4 is used. The colour plot is the non-axisymetric part of the vorticity, it is concentrated around the position of the bump. The black lines are the streamlines of the non-axisymetric part of the velocity.

\section{Conclusion}

The simulations presented here have proved that the RWI can develop in a 3D accretion disc at the extremum of specific vorticity. The radial and vertical structure are in agreement with the RWI linear theory. Now that the 3D structure of the instability known, we now aim to perform simulation of the RWI growing from the disc inner edge where the HFQPO originates.

\section{References}

[1] Li, H., Finn, J.M.., Lovelace, R.V.E., Colgate, S.A., 2000, ApJ, 553, 1023

[2] Li, H., Colgate, S.A., Wendroff, B., Liska, R., 2001, ApJ, 551, 874

[3] Lovelace, R.V.E, Li, H., Colgate, S.A., Nelson, A.F., 1999, ApJ, 513, 805

[4] Tagger, M. \& Varniere, P., 2006, ApJ, 652, 1457 